\section{DE RHETORICA HISTORIAQUE: UN EXAMEN DES RAPPORTS ESSENTIELS ET FONCTIONNELS ENTRE LES DEUX DISCIPLINES CHEZ LES RHÉTEURS GRECS}

\begin{abstract}
Rui Miguel Duarte*
RESUMO: Retórica e história: o que têm uma a ver com a outra? Eis uma questão que pode suscitar surpresa. Com efeito, trata-se aparentemente de dois diferentes géneros de discurso, nos seus objectos como propósitos: a retórica é o discurso que visa a persuasão e os possíveis, ao passo que a história narra eventos reais; a primeira emite um ponto de vista, uma opinião da qual se pretende convencer outrem, enquanto a história relevaria mais do domínio do objectivo. Todavia, as coisas não seriam assim tão estritas, antes as duas disciplinas poderiam ser complementares e mutuamente auxiliares. As relações existentes entre uma e outra seriam principalmente de natureza utilitária. A retórica é útil como treinadora de estratégias e técnicas que servem para a composição literária, coinclusão do género histórico. Em sentido contrário, a história é fornecedora de matéria e temas para os debates e os discursos da retórica, os quais, se por um lado respeitam os dados daquela, por outro deles se servia, manipulando-os, para os estritos propósitos de compor declamações engenhosas. O presente artigo examinará o estado da arte do problema entre os retores gregos.
\end{abstract}

PALAVRAS-CHAVE: história; retórica; rétores gregos; relações funcionais.

\section{DE RHETORICA HISTORIAQUE: A SURVEY OF THE ESSENTIAL AND FUNCTIONAL RELATIONSHIPS BETWEEN THESE TWO FIELDS IN GREEK RHETORS}

* Investigador Integrado do Centro de Estudos Clássicos (CEC), Faculdade de Letras, Universidade de Lisboa.

\footnotetext{
ABSTRACT: Rhetoric and history: what have each of them to do with the other? That is a question that can cause some surprise. In fact, they are apparently two different kinds of discourse, considering both the subjects each of them deal with and their goals: rhetoric is the discourse that aims at persuasion and possibilities, whereas history recounts real facts; the former puts forward point of view, an opinion someone should be persuades of, while history would be mainly objective. Nevertheless, things could not be so strict in themselves; these two matters could be
} 
rather complementary and mutually useful. The relations they had to each other were mainly utilitarian. Rhetoric is useful as a trainer of strategies and techniques of literary composition, including of the history. On the contrary, history provided subjects and themes to debates and rhetorical discourses, which on the one hand respected the historical data, but on the other hand also used and manipulated them pour the purposes of ingenious declamations. This paper shall address the state of the art of the problem in Greek rhetoric.

KEYWORDS: history; rhetoric; Greek Rhetors; functional connections.

\section{Préambule}

$\mathrm{R}$

hétorique et histoire: qu'ont-elles à voir l'une avec l'autre ? La question ainsi posée, à l'improviste, pourrait surprendre. Apparemment il s'agit de deux discours si abord, au moindre rapport entre elles. En effet, la rhétorique, classiquement entendue, est le discours (ce mot étant pris dans un sens plus large) qui vise la persuasion dans les affaires publiques (ayant donc un versant pragmatique), alors que l'histoire raconte des évènements. Ce versant pragmatique est bien présent dans des définitions et conceptions anciennes. Pensons, à titre d'exemple, à l'une des définitions de la rhétorique transmises par les Grecs,

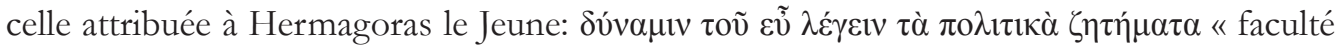
de bien parler des questions politiques $\gg .^{1}$

Aristote, en Poétique IX 2, 1451 b, écrit que les différences entre le discours du poète et celui de l'historien ne consistent pas en la forme métrique ou non-métrique dont ils se présentent (donc, en des aspects formels). La preuve en est que l'œuvre d'Hérodote, même si elle était réécrite en vers, n'en serait pas moins de l'histoire. La différence réside ailleurs:

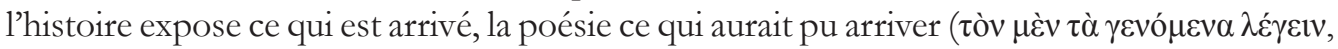

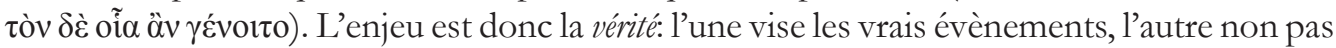
les faits tel quels, mais des choses qui pourraient l'être. Or, la rhétorique reflète et se pose ce même problème. Le Stagirite, essayant de la délivrer du malaise causé par les critiques faites à la pratique sophistique, assume la séparation des domaines et des buts, la rhétorique ne visant pas la vérité, mais ce qui est vraisemblable et qui peut être le plus souvent. C'est là le

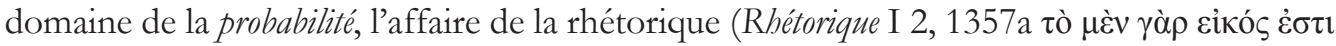

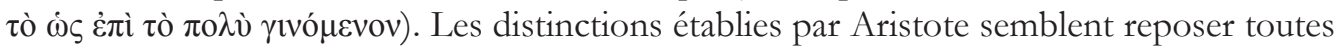
sur les mêmes principes d'examen: la notion de réel évènementiel s'opposant à ce qui ne pourrait être qu'une probabilité.

Le thème est susceptible de traitements et d'examens divers, en des époques et lieux également divers. Dans les pages qui suivent, on se propose d'examiner quelques réflexions apportées par des théoriciens grecs, notamment ceux de l'époque impériale. ${ }^{2}$ Quant aux

\footnotetext{
${ }^{1}$ T3 Woerther (2012).

${ }^{2}$ Charmes Guérin (2009), «La persona oratoire entre rhétorique, biographie et histoire. Le cas des Controuersia de Sénèque le Rhéteur », Interférences 5 analyse les structures rhétoriques d'interprétation
} 
méthodes suivies, il faut mettre en évidence comme particulièrement utile une recherche menée sur le TLG-E $\mathrm{E}^{3}$ de textes appartenant au genre " Rhet » avec comme mots-clefs

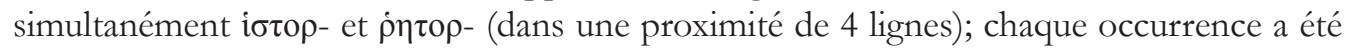
examinée afin de détecter celles où il était question des rapports entre rhétorique et histoire. Triage fait, les occurrences importantes ont été rangées en fonction de différents types de rapports détectés. Voici le résultat de cet examen.

\section{LA RHÉtORIQUE AU PROFIT DE L'HISTOIRE}

A l'époque impériale la rhétorique était déjà établie à la fois en tant que fournisseuse de recettes et qu'entraîneuse de stratégies et techniques servant à la composition littéraire. Dans le premier des deux chapitres introductifs de ses exercices préparatoires ( $\left.\pi \rho \gamma_{\gamma} \mu v \alpha \sigma \mu \alpha \tau \alpha\right)$, Aélius Théon (Patillon, 1997, 60.1-8, 2), maître du premier siècle de notre ère, le déclare expressément, à propos de l'utilité de ces exercices pour le développement aussi bien de la compétence oratoire que de la littéraire:

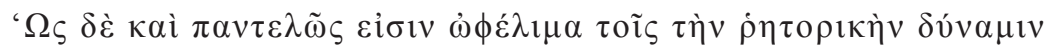

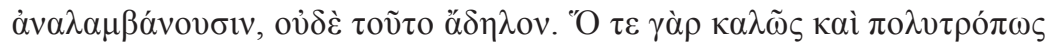

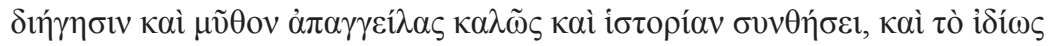

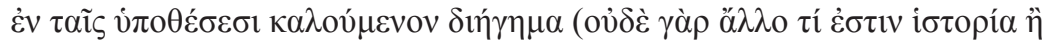

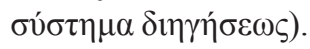

Qu'ils sont tout à fait utiles pour l'acquisition de la maitrise de l'art oratoire, cela est également évident. En effet l'art de présenter comme il faut et sous des formes diverses un récit ou une fable, implique l'art de composer une histoire et ce qu'on appelle la narration dans les causes: car une histoire n'est rien d'autre qu'un ensemble de récits.

Le socle des liaisons de la rhétorique à l'histoire est la procédure du récit, ou narration oratoire ( $\left.\delta \eta_{\gamma} \eta \mu \alpha\right)$. En effet, il est l'une des parties canoniques du discours dans le genre judiciaire, celle où l'on raconte les faits devant les juges (voir la description technique et théorique par Lausberg, 1960, \289-347). Ainsi l'utilité de la rhétorique pour l'histoire devient claire. Rhétorique et histoire étaient tellement et si étroitement liées que l'histoire

mises en place par Sénèque le Rhéteur dans ses Contronersiae au sujet des caractéristiques des déclamateurs. Le Rhéteur vise à préserver des exemples valides du passé. L'auteur conclut que le Rhéteur " inscrit son propos dans l'histoire », et aussi qu'il « fait des caractéristiques des différents déclamateurs le produit d'une modification historique ». Par ce biais, la rhétorique devient chez Sénèque plus critique que descriptive. Ici, on le voit, c'est le caractère paradigmatique et de modèle pour la rhétorique qui est en cause. Les deux perspectives vont de pair et se trouvent liées. L'étude des rapports des deux disciplines est, semble-t-il, un champ de recherche à explorer davantage, tant dans le monde grec que dans le romain.

${ }^{3}$ Nous avons eu recours au logiciel d'interrogation SNS Greek \& Latin de la Scuola Normale Superiore de Pisa sur Mac OS X. 
n'est qu'un ensemble organisé ( $\sigma u ́ \sigma \tau \eta \mu \alpha)$ de récits, et que narrer les faits mis en examen devant un tribunal était formellement la même chose que narrer des évènements. Quant au genre littéraire de l'histoire, la rhétorique, par le biais de l'entraînement de l'habileté de narrer, joue un rôle fonctionnel. La conséquence de la pensée théonienne est que la maitrise de procédures rhétoriques habilite techniquement à l'écriture de l'histoire.

Ceci est plus évident si l'on pense à l'exercice de la chrie, celui qui semble être le point de rencontre le plus important des deux disciplines. Définie comme une assertion ou une action succincte, avisée et ayant de la grâce, attribuée à tel personnage, ${ }^{4}$ la chrie ( $\left.\chi \rho \varepsilon i ́ \alpha\right)$ est la façon la plus élémentaire de transmission de mémoire historique, de paroles et d'actes des personnages historiques.

La chrie étant naturellement succincte, peut cependant être étendue. On a alors un

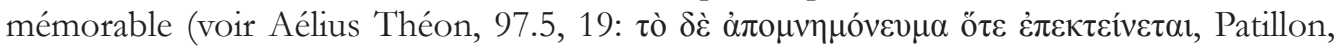
1997). ${ }^{5}$ Au sein des écoles de rhétorique élémentaire on s'exerçait aussi bien à amplifier une chrie qu'à l'opération contraire (ib. 103.30-33, 27-28). L'allongement se fait par des procédures techniques telles que le développement des dialogues, de l'action et du pathos. Soit par exemple une chrie d'Epaminondas, général thébain (ib. 103.33-104.2): 'E $\pi \alpha \mu \varepsilon ı \omega ́ v \delta \alpha \varsigma$,

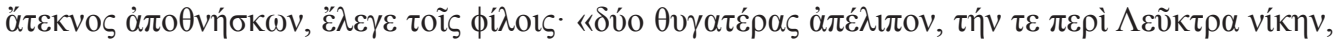

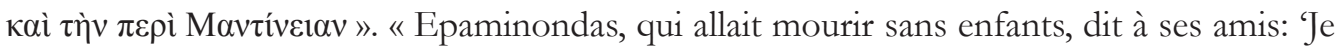
laisse deux filles, la victoire de Leuctres et celle de Mantinée ». Et voici comment Théon l'allonge (de façon largement créative, par ailleurs):

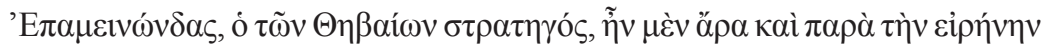

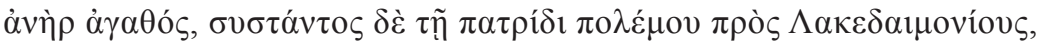

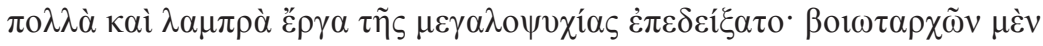

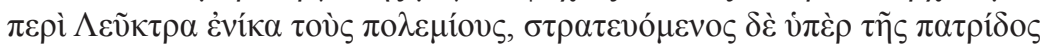

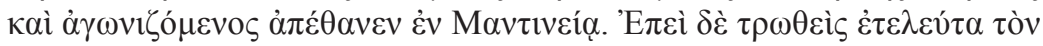

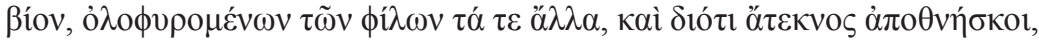

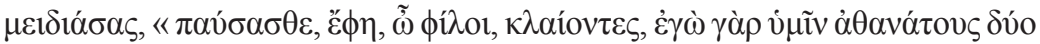

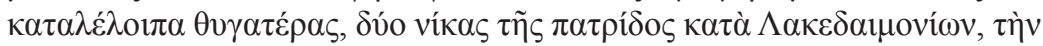

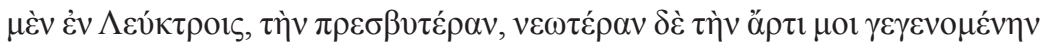

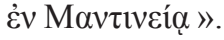

Epaminondas, général thébain, avait déjà montré sa valeur en temps de paix; et lorsque sa patrie entra en guerre avec Lacédémone, il donna beaucoup d'exemples éclatants de sa grandeur d'âme: après avoir vaincu les ennemis à Leuctres en qualité de béotarque, c'est en qualité de soldat qu'il se battit pour sa patrie à Mantinée et qu'il y mourut. Alors qu'il était blessé et mourant, et que ses amis gémissaient sur son malheur et en particulier sur le fait qu'il mourait sans enfants, il dit en souriant: « séchez vos larmes, mes amis, je vous laisse deux filles immortelles, deux victoires de notre patrie

\footnotetext{
${ }^{4}$ Cf. la définition par Aélius Théon cap. 3 (Patillon, 1997: 96.19-21, 18. Voir aussi les définitions du Pseudo-Hermogène (Patillon, 2008a: III 1) et d'Aphthonios (ib. III 1).

${ }^{5}$ Rappelons-nous de l'œuvre de ce nom de Xénophon.
} 
contre Lacédémone, celle de Leuctres, l'aînée, et la cadette nouveau-née, de Mantinée.

La chrie est prise comme modèle pour façonner une petite narration d'épisodes de vie avec des intentions et structures argumentatives. Littérairement elle devient un récit.

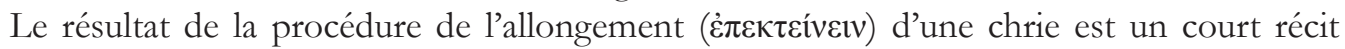
biographique sur un personnage historique.

Nonobstant, un tel rôle fonctionnel de la rhétorique vis-à-vis de l'écriture de l'histoire ne se limite pas à la seule et stricte composition des récits d'actions (ib. 66.31-67.30, 10-11). L'historien peut mettre en scène des procédures qui sont typiquement oratoires, tels que: les

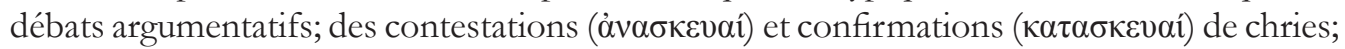

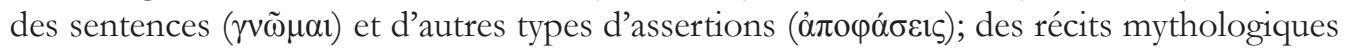
(l'exercice du $\mu \tilde{v} \theta$ os) et d'autres réels $(\delta \eta \eta \gamma \eta ́ \mu \alpha \tau \alpha)$. Les professeurs ainsi que les élèves avaient à leur disposition un patrimoine varié et prêt-à-porter de genres, textes et auteurs comme modèles de l'emploi des exercices. Parmi les auteurs, Théon cite des historiens: Ephore (66.27; 67.3 , 9, 20; 69.32; 71.23; 95.24); Hérodote (66.9, 23, 25; 67.6, 14; 68.12; 69.31; 83.5; 84.4; 86.24; 91.28; 93.11; 95.12; 118.15; 120.5; 116.7); Thucydide (63.14, 24: 66.23, 24; 67.17; 68.8, 26; 69.32; 70.13; 80.16; 82.21: 84.4, 28; 86.19; 84.4, 28; 86.19; 87.22; 90.21; 93.8; 118.25; 119.4; 110.15); Théopompe (63.16; 66.11, 21; 67.22; 68.14, 27; 70.6; 81.1; 110.33). ${ }^{6}$

Le portrait fait par Aélius Théon de cette éducation rhétorique élémentaire est celui de modalités variées d'exercices en complexité croissante et qui entraîne l'élève dans la maîtrise de l'éloquence, du raisonnement et de l'écriture. On peut facilement comprendre que tous, orateur, auteur de fictions ou historien, passaient par les mêmes formations et qu'ils devenaient aussi compétents pour se lancer dans la carrière oratoire que dans celle d'historien, lorsque les rudiments de l'art avaient été acquis. La rhétorique fait fonction de pierre angulaire de la littérature, aussi bien pour tout écrivain comme pour tout lecteur. C'est exactement ce que le technographe écrit, dans sa réflexion à propos de l'utilité des exercices, citée plus-haut. Quelques lignes plus bas (60.19-27, 2-3), il écrit que, par exemple, l'utilité de ce qu'on appelle le lieu et la description se voit, respectivement, dans l'histoire et l'éloquence; l'exercice de la prosopopée est utile pour toutes deux et aussi pour les genres du dialogue et de la poésie. En outre, l'importance de leur pratique est montrée d'une manière générale dans un cadre plus large de situations: « dans les conversations de la vie courante » Kỏv $\tau \tilde{\omega}$

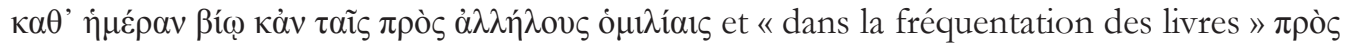

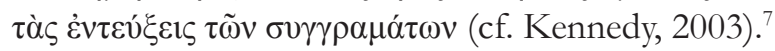

\footnotetext{
${ }^{6}$ On est dans la période connue par Seconde Sophistique. Parmi les auteurs plus représentatifs de cette période et de la rhétorique, il faut rappeler l'un des plus renommés, Plutarque. Son style dénonce

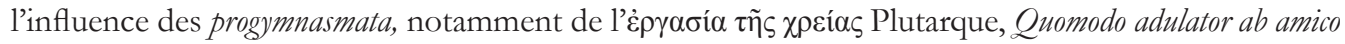
internoscatur 70e-71e; Quomodo quis suos in virtute sentiat profectus 78e-79d; De fraterno amore 490d-491d; De tranquilitate animi $474 \mathrm{~d}-475 \mathrm{c}$.

${ }^{7}$ Dans ce volume se trouvent réunies les traductions des traités d'Aélius Théon, Ps.-Hermogène, Aphthonios, Nikolaos, du commentaire au traité d'Aphthonios attribué à Jean de Sardes et des
} 
Pensons par ailleurs aux rapports que Rufus de Périnthe, vers la deuxième moitié du II s. après J.-C. (Patillon 2001: 238), établit entre les deux. En effet, aux trois genres aristotéliens traditionnels de la rhétorique (judiciaire, délibérative et encomiastique) ce rhéteur (ib. 2.2 et 6-7) en ajoute un quatrième, l'historique. Il ne développe pas ce point, mais en fournit un début d'explication: la rhétorique (2.6) enseigne à narrer de façon ornée les actions passées

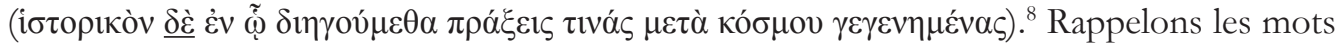
de la Rhétorique d'Aristote au sujet des actions passées. L'allusion de Rufus ne concerne pas, apparemment, la narration strictu sensu dans les discours, appartenant surtout au genre judiciaire, car cette narration en est une des divisions. Il semble penser à la narration de faits en tant que genre autonome, c'est-à-dire, à l'histoire. Ce qui fait de lui un genre rhétorique, c'est l'ornementation. Les trois autres genres, les traditionnels, concernent les débats et les discours prononcés en public. L'historique, lui, présente un caractère définitivement plutôt littéraire, quoique redevable des ressources de la rhétorique, non de ceux relevant de l'argumentation, mais de ceux touchant au seul côté strictement formel, c'est-à-dire, stylistique, du discours.

Rufus n'est pas le seul à mentionner ce quatrième genre, Syrianus nous rapportant qu'elle se trouvait chez certains anciens théoriciens. (Rabe, 1893: 11.16-17 oi $\delta \dot{\varepsilon} \tau \varepsilon \dot{\varepsilon} \tau \alpha \rho \tau o v$

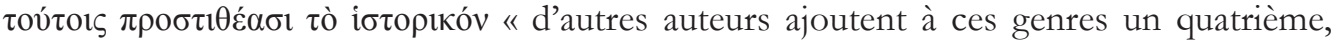
l'historique ». Syrianus pense peut-être ici à Rufus. Quoi qu'il en soit, Rufus est le seul témoin de cette tradition (cf. Patillon, 2001: 247). Remonterait-elle à Aristote? C'est l'avis d'un

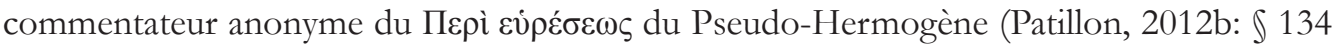

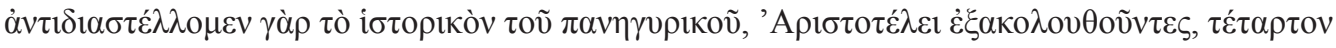

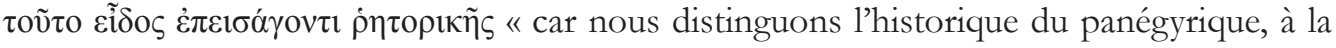
suite d'Aristote, qui introduit ce dernier comme quatrième espèce de la rhétorique ${ }^{9}$ ) et Nikolaus de Myra, Progymnasmata 55.8 Felten.

Or, Nikolaos (ib. 12.17-13.13), après avoir distingué trois espèces de narration (mythiques, historiques et pragmatiques ou politiques), établit une autre différenciation: les récits historiques racontent les évènements passés, les politiques sont ceux que les orateurs utilisent dans les procès. Il y a là une distinction entre les narrations dans les discours et celles du genre historique propre. C'est une précision qui prend en compte des différences de genre littéraire.

fragments du traité attribué à Sopatros. Dans l'introduction, Kennedy résume bien le triple caractère de cette rhétorique — l'entraînement dans l'art de l'éloquence, la formation morale et la letteraturizazzione (ix): «The curriculum described in these works, featuring a series of set exercises of increasing difficulty, was the source of facility in writing and oral expression for many persons ad training for speech in public life. In addition, the composisitons inculcated cultural values, as well as understanding of conventional literary forms for those who entered on literature as a career or as an elegant pastime. »

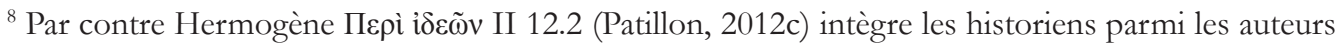
panégyriques, le troisième genre. Plus bas (à partir du \ 15) il traite séparément le style des historiens. ${ }^{9}=$ Walz, 1832-1836: VII 794.6-9. De ce que nous connaissons de l'œuvre conservée d'Aristote, aucun passage ne correspond à telle attribution. Cf. encore Cicéron Or. 207. 


\section{L'HISTOIRE AU PROFIT DE LA RHÉTORIQUE}

En sens inverse, l'histoire peut servir aux besoins de l'éloquence et de l'élaboration des arguments. C'est à nouveau un rôle fonctionnel celui que la discipline auxiliaire joue. Néanmoins, dans les cas où ce rôle appartient à l'histoire, c'est la question même du bon droit et de la conformité des débats et des discours qui se pose.

Notre examen se fera selon trois approches: l'histoire en tant que fournisseuse d'exemples et ressources pour les arguments; la crédibilité historique en tant que fondement sur lequel s'étaye la crédibilité même de la rhétorique; le dernier, celui des manipulations de l'histoire par la rhétorique.

La première approche de la question n'est pas inédite. Selon Aristote, Rhétorique I 4, 1360a, l'orateur est tenu de maitriser une variété de matières et de domaines; parmi eux, les lois et les mœurs de plusieurs peuples, ainsi que les écrits des historiens. La raison est qu'il en aura besoin pour ses discours. Cependant, Aristote même introduit une restriction: tout cela appartient à la science politique, non pas à la rhétorique. Et cela d'autant plus que, à son avis (Rhétorique I 2, 1355b), la rhétorique ne possède pas d'objet particulier. Le livre de Nouhaud (1982), sur l'usage de l'histoire par les orateurs attiques des $V^{e}-I V^{e}$ siècles av. J.-C., est un éclatant exemple de cette approche. D'après les conclusions de l'auteur, l'histoire chez ces orateurs servait comme une ressource pour illustrer des points de l'argumentation.

Aélius Théon d'Alexandrie, environ quatre siècles plus tard, donne un conseil pareil: l'histoire est une source de beaux exemples. Ceci est bien clair, notamment, dans

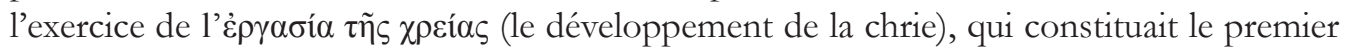
essai d'élaboration d'une pièce oratoire entière, en huit parties, dont le thème était proposé

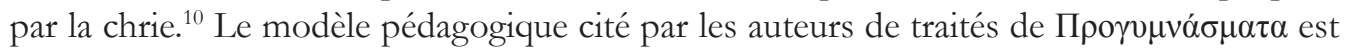

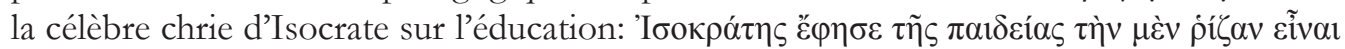

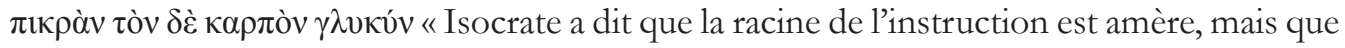
son fruit est doux $»)$. L'exemple $(\pi \alpha \rho \alpha ́ \delta \varepsilon(\gamma \mu \alpha)$ — la sixième partie de la pièce — donné par le

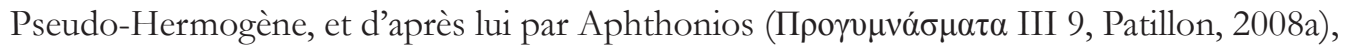
est Démosthène. Les enjeux sont la qualité du personnage historique et les éléments connus de sa biographie. Le traitement qu'en donne le Pseudo-Hermogène est sommaire (ib. III

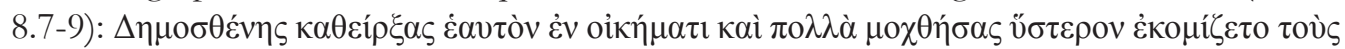

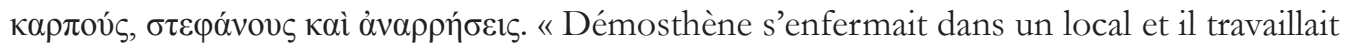
dur, mais plus tard il en a récolté les fruits, couronnes et proclamations ». Aphthonios, lui,

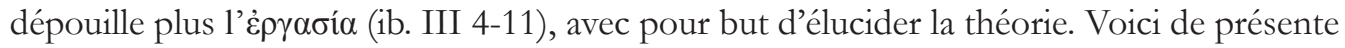
l'argumentation par l'exemple (ib. III 9):

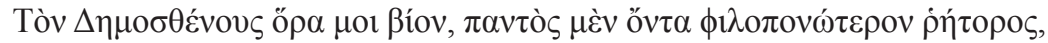

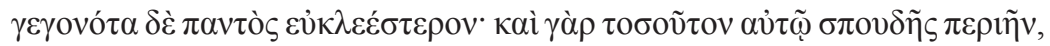

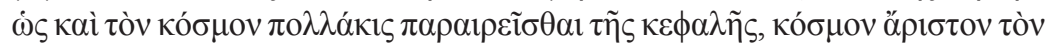
$\dot{\varepsilon} \xi \dot{\alpha} \rho \varepsilon \tau \tilde{\eta} \varsigma \dot{\eta} \gamma \eta \sigma \alpha ́ \mu \varepsilon v o v \cdot$

\footnotetext{
${ }^{10}$ Voir Alexandre Júnior (1989); Ronald F. Hock \& Edward N. O’Neill (1986).
} 
Voici donc la vie de Démosthène: aucun orateur n'en a eu de plus laborieuse, mais aucun non plus de plus illustre. Il poussait l'ardeur si loin qu'il allait souvent jusqu'à supprimer l'ornement de sa tête, considérant que le plus bel ornement est celui de la vertu. ${ }^{11}$

La tradition transmet de Démosthène la mémoire d'un homme qui, ambitionnant la gloire oratoire, s'est soumis à une discipline sévère. Plutarque, historien et biographe, ajoute des détails (Dém. 7): Démosthène a construit un cabinet de travail souterrain où il se rendait chaque jour et s'entraînait à la déclamation, la moitié de la tête rasée, y passant même deux ou trois mois d'affilée sans quitter les lieux, pour ne pas avoir à souffrir la honte d'être vu en de telles conditions. L'histoire, ou ce qu'on croyait être l'histoire, sert les propos de la rhétorique: le grand orateur est l'exemple de la réussite à laquelle les sacrifices et les efforts peuvent conduire; l'histoire se porte caution de l'argumentation et valide le point de vue qu'on se propose de démontrer.

Le Pseudo-Hermogène du traité sur l'invention (Patillon, 2012a: II 4, 7), dans son

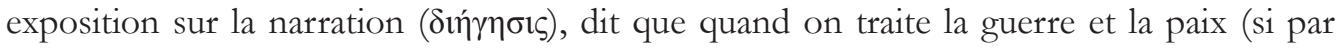
exemple on veut arrêter une guerre), dans la préexposition préalable à la narration on pourra ajouter les raisons que l'histoire met à notre disposition, si elles existent; de même, plus bas, au sujet de l'impiété et du meurtre (II 5.2,4-6). ${ }^{12}$

Que l'orateur utilise les ressources de l'histoire suscite, pourtant, le désaccord du Pseudo-Aélius Aristide (Patillon, 2002b: II 25.1-9). Ce maittre reproche aux orateurs politiques le fait d'ajouter dans les discours simples des idées prises de l'extérieur et hors sujet. C'est là, constate-t-il, une pratique habituelle. L'histoire est l'une des sources de ces idées. Il faudrait, selon le conseil du rhéteur, le faire en s'en tenant au sujet du discours. Outre la critique de certaines pratiques chez les orateurs, on retient de ces paroles le fait que les données et les évènements historiques n'étaient pas étrangers à l'activité des orateurs et que ceux-ci les connaissaient et les citaient à titre d'exemples illustratifs. Cette mention est répétée presque littéralement plus bas (II 104). Au livre I (I 132.1-4; Patillon, 2002a), à propos de la douceur ( $\pi \varepsilon \rho i ̀ ~ \gamma \lambda v \kappa v ́ \tau \eta \tau o \varsigma)$, le rhéteur dit que cette vertu se tire de trois facteurs, parmi lesquels la pensée. Les emprunts à l'histoire, aux proverbes ou aux fables procurent la douceur de la pensée. Plus bas (Patillon, 2002b: I 175), un exemple d'un exercice de déclamation est fourni. Après une deuxième amplification à partir de l'acte, il y a lieu à employer les données de l'histoire.

Une ébauche de notre deuxième approche des rapports entre les deux disciplines se trouve dans le passage Poétique IX 2 1451b d'Aristote, cité dans l'introduction de notre étude, au sujet des différences entre le discours historique, et le poétique. Rappelons-le: il est question d'une distinction centrale entre la réalité événementielle et ce qui reste dans

\footnotetext{
${ }^{11}$ Libanios, dans ses Progymnasmata 92.10-93.3, explique que Démosthène agissait ainsi, maltraitant la tête, pour s'obliger à rester là où il travaillait, et plus tard il a pu voir sa tête couronnée. Les romains aussi gardent cette tradition (voir Quintilien 11.3.54).

${ }^{12}$ Ce recours est à rapprocher de l'usage des anciens orateurs (Patillon, 2012: 25 n. 120; Nouhaud, 1982).
} 
le domaine du possible; en d'autres mots, le vrai et vraisemblable. Il y a ici deux plans isotopiques importants contribuant à la compréhension de ce qui fait de la rhétorique ce

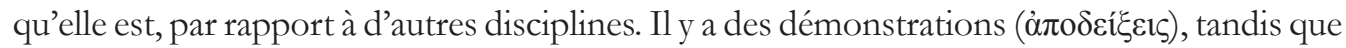
la rhétorique emploie des enthymèmes $(\dot{\varepsilon} v \theta v \mu \eta \dot{\mu} \mu \alpha \tau)$, qui sont une sorte de démonstration (Rhétorique I 1 1355a). Il y a, en d'autres termes, le vrai ( $\tau$ ò $\dot{\alpha} \lambda \eta \theta \dot{\varepsilon} \zeta$ ) et le vraisemblable (ib.

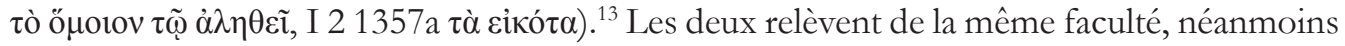
ils présentent une dissimilitude. Les enthymèmes (la démonstration en rhétorique) se tirent des propositions (ib. 1357a), lesquelles peuvent être nécessaires ou pour la plupart simplement

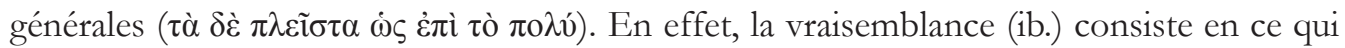

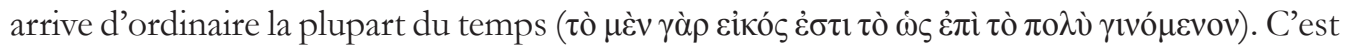
l'affaire de la rhétorique. Voici les données essentielles de la question.

Au cœur des rapports entre rhétorique et histoire, notamment dans la mesure où celle-ci sert les propos de la première, on rencontre la vraisemblance. Elle est l'une des vertus du $\delta ı \eta ́ \gamma \eta \mu \alpha$ (narration ou récit). Les données et les contraintes de l'histoire légitiment, arcboutent ou, tout au contraire, empêchent et rendent impossible l'exercice de la rhétorique? Aélius Théon (79.28-32, 40, Patillon, 1987) se prononce ainsi sur cette vertu et son apport au discours:

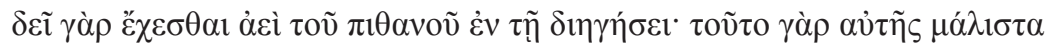

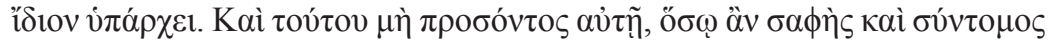

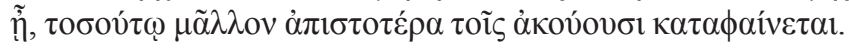

Car il faut toujours s'attacher à la vraisemblance dans le récit: c'est là en effet sa qualité première. En est-il dépourvu, plus il sera clair et concis, moins il paraîtra crédible aux auditeurs. ${ }^{14}$

Le défaut de vraisemblable risque de faire pencher la balance des vertus vers le côté des deux autres. Il faut donc, poursuit-il dans les lignes suivantes, les utiliser en doses équilibrées. Cependant, la vraisemblance est la primordiale.

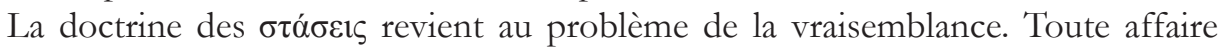
susceptible d'être mis en examen se composant d'actes et personnes (Hermogène, Patillon, 2008b: 4.1-2), le problème de la vraisemblance se pose d'abord au sujet de l'une des espèces

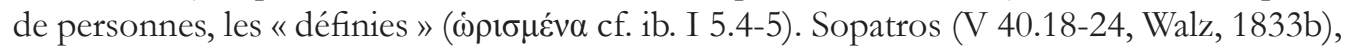

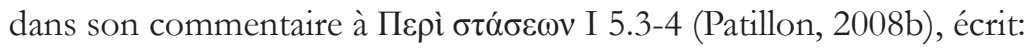

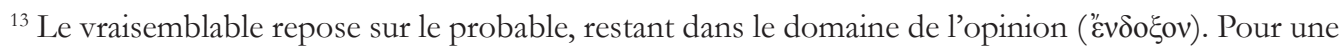
définition cf. Arist. Top. 1.1.

${ }^{14} \mathrm{Il}$ faut noter que ce pédagogue utilise de façon interchangeable les deux mots désignant en grec

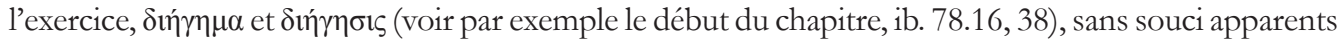
de distinction ni de précision terminologique. Les théories des vertus du récit (dont les deux autres sont la clarté et la concision) remonteraient au moins à la Rhétorique à Alexandre (1438a 19b 15), comme le rappelle Patillon, et se sont maintenues en dépit de quelques fluctuations (1997: 140). Quintilien (4.2.11) fait remonter la notion à Isocrate et son entourage.
} 


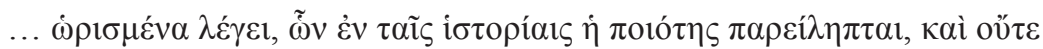

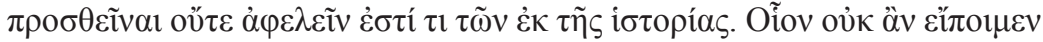

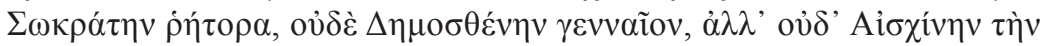

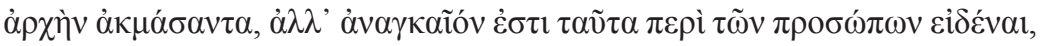

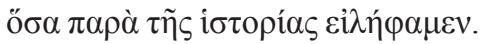

Il (scil. Hermogène) appelle définies les personnes dont la qualification dépend de l'histoire, sans qu'on puisse rien ajouter ni supprimer de ce qui relève de l'histoire. Par exemple, on ne saurait dire que Socrate est un orateur, ni que Démosthène était un homme noble, ni qu'Eschine a atteint le sommet du pouvoir, mais il est impératif de connaître tout ce que l'histoire met à notre disposition au sujet des personnes.

Ce problème se pose aussi à l'égard d'un autre type de personnes, désigné par des noms propres (I 1.5.4-5 кúpı $\alpha$, Patillon, 2008). Sopatros (V 40.24-29, Walz, 1833b) commente:

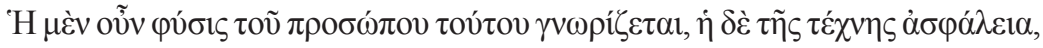

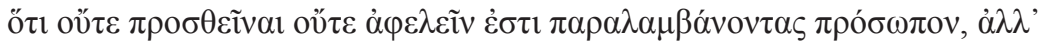

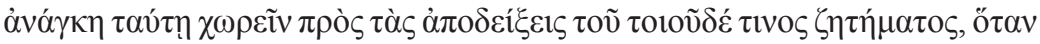

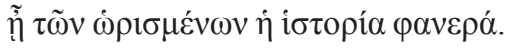

La nature de la personne, donc, est reconnue d'après ces choses. Quant à l'art, la sûreté de sa technique vient de ce qu'il n'est pas possible, quand on prend une personne, ni d'ajouter ni de supprimer, mais qu'il faut en passer par là pour les démonstrations de telle ou telle question, quand l'histoire des personnes définies est claire.

L'Anonyme P (1.32.7-8, Duarte, 2006: = VII 118.24-26, Walz, 1832-1836), en

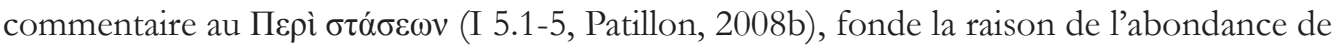
ressources pour le discours chez ces deux types de personnes sur les données de l'histoire: Tò

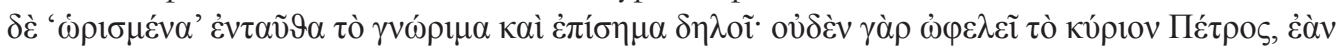

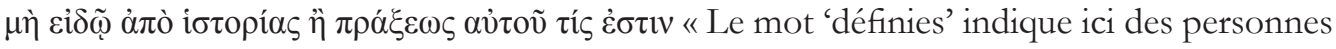
'connues' et 'remarquables', puisque le nom propre ne sert à rien (par exemple, 'Pierre'), si l'on ne sait pas, d'après l'histoire et ses actes, qui il est. ${ }^{15}$ Comme son devancier Sopatros, ce commentateur se montre insatisfait de la formulation hermogénienne des deux types de personne: les définies, d'autant que celles désignées par des noms propres, n’ont en ellesmêmes aucune force de par leurs seuls noms, faute de tout ce qu'on peut prédiquer d'elles et de leurs actes. En un mot, sans l'histoire.

\footnotetext{
${ }^{15}$ Dissertation de Doctorat, comprenant une édition critique partielle, traduction en portugais et

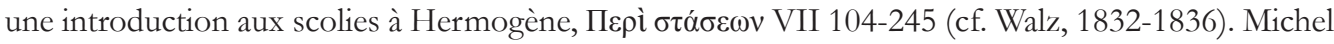
Patillon nous a gentiment transmis que des recherches faites récemment par ses soins l'ont amené à croire qu'une grande partie de ces commentaires sont dus à Eusthate. Ces recherches aboutiront, nous a-t-il confié, à une nouvelle édition de ce commentateur, où la question de l'auteur sera débattue.
} 
Hermogène, plus bas, traite les huit espèces de problèmes inconsistants (I 14-22, Patillon, 2008b). Parmi elles, l'invraisemblable, qui occupe le cinquième rang (ib. I 18):

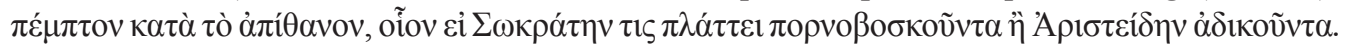
"Cinquièmement la question invraisemblable, par exemple si l'énoncé fait de Socrate un tenancier de maison close ${ }^{16}$ ou d'Aristide un criminel. » Dans son commentaire à ce passage, Sopatros remarque que la question de l'invraisemblance ne se poserait pas, si par exemple $(\mathrm{V}$ 66.24-67.1, Walz, 1833b) le chef d'accusation contre Socrate était l'impiété - c'était celle-là l'accusation qui l'a fait condamner à mort -, ou toute autre sauf l'immoralité. Si c'était le cas, on aurait là une question susceptible d'examen. Quant à Aristide, de même, on aurait une question bien formée s'il était accusé de désertion ou d'arrogance. Ou encore, si on accusait Achille de trahison ou de se laisser corrompre, mais jamais de lâcheté ni de désertion; ou Ajax de conspiration, de témérité ou d'avoir prémédité la destruction du campement, mais non pas de lâcheté ni de désertion. De tels problèmes seraient consistants. Car, comme le commentateur conclut: la réputation et la qualité d'une personne repose sur une certaine vertu, et il n'y a d'invraisemblance que lorsqu'on accuse cette personne de la chose contraire

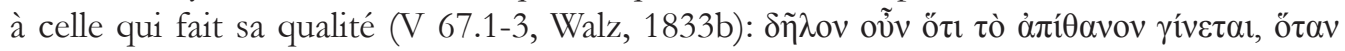

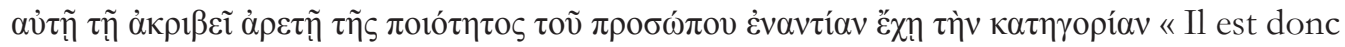
évident qu'il y a une question invraisemblable lorsque l'accusation contredit la vertu même qui fait précisément ce que la personne est par sa qualité. »

Le commentateur Marcellinus (IV 158.25-30, Walz, 1833a) explique ainsi une telle hypothèse d'écart de conduite chez Socrate et Aristide:

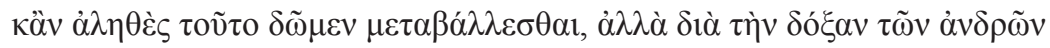

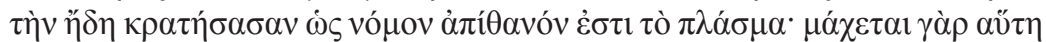

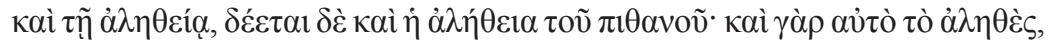

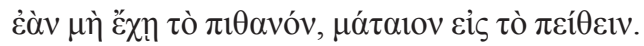

Même si l'on admet comme vrai qu'ils se sont égarés, à cause de la réputation de ces hommes, qui a gagné désormais statut de loi, la fiction est invraisemblable. En effet, la réputation contredit la vérité et la vérité a besoin de vraisemblance, car la vérité elle-même, si elle est dépourvue de vraisemblance, ne sert en rien à la crédibilité.

On a donc une question invraisemblable si l'énoncé est en contradiction avec les données extralinguistiques de l'histoire et du monde référentiel. En effet, Socrate est un exemple traditionnel de vertu et sagesse, et Aristide d'honnêteté et justice. L'éthos de la personne est le socle de sa réputation. Celle-ci a la force d'une loi: elle oblige la communauté des participants du débat rhétorique - orateurs et auditeurs, à qui il appartient de juger - en leur imposant certaines contraintes au sujet de ce qu'il faut et ne faut pas accepter comme défendable dans le débat. C'est grâce à l'histoire, à ce qu'on peut connaitre de la vie de ces personnages, qu'ils deviennent des modèles référentiels. Ces modèles «n’appartiennent pas

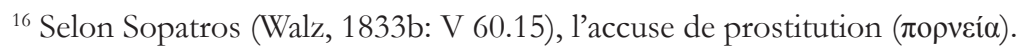


seulement au contexte immédiat, à la situation où se trouvent les interlocuteurs, mais à tout ce qu'ils savent du monde. » (Patillon, 1988: 27 n. 3). Marcellinus tranche encore: une chose est la vérité (les faits), celle-ci ne suffisant pourtant pas; une autre est la vraisemblance, le corpus de connaissances que l'univers des participants du débat partage au sujet des personnages et des évènements historiques. Dans la rhétorique, la vraisemblance, qui se fonde sur les données incontournables de l'histoire, est une exigence si forte qu'elle vient à l'aide de la vérité même, celle-ci ne suffisant pas par elle-même à rendre le discours acceptable; il faut qu'elle soit acceptée et agréée en tant que telle par la communauté. La question est donc: est-ce que cela est conforme à ce qu'on connaît de la personne, que l'énoncé du problème en donne une image qui contredit sa réputation et ses mœurs? ${ }^{17}$ Si c'est le cas, il ne passera pas en principe le test de la vraisemblance et sera refusé par les auditeurs.

Le problème de la vérité se pose de façon encore plus aigüe dans une autre espèce des $\dot{\sigma} \sigma u ́ \sigma \tau \alpha \tau$, laquelle, dans le rangement hermogénien, suit la vraisemblable, au sixième rang. C'est la question frappée par le vice d'impossibilité. Dans de tels cas, le seul problème qui se pose n'est pas de savoir si une version de l'histoire est compatible avec ce que l'on connait de certains personnages, mais que telle version de l'histoire est contredite par l'histoire même. Les choses se sont passées autrement. Le degré d'inconsistance de cette espèce de questions

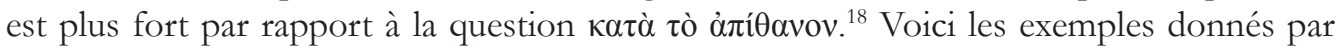

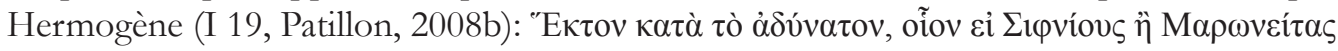

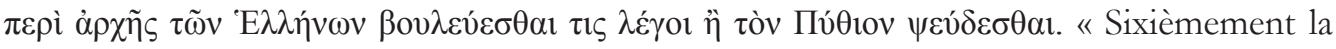
question impossible, par exemple si l'on dit que les habitants de Siphnos ou de Marônéia forment le dessein de commander aux Grecs ou qu'Apollon ment. ». En quoi ces exemples sont-ils impossibles? Passons à côté du deuxième exemple et concentrons l'examen sur celui où il y a un cas d'histoire, la question de la place de Siphnos et Marônéia dans le jeu des puissances hellènes.

Certains des commentateurs d'Hermogène pensent que sa théorie ici est

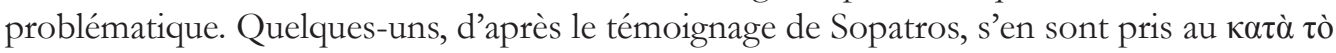

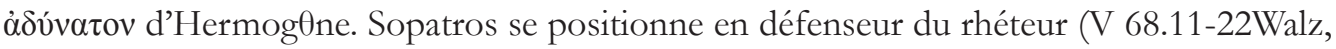
1833b):

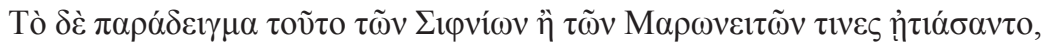

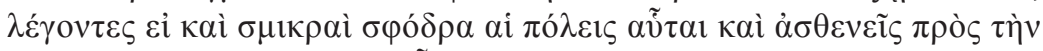

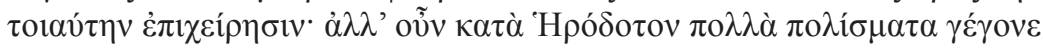

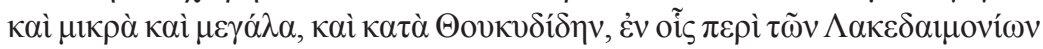

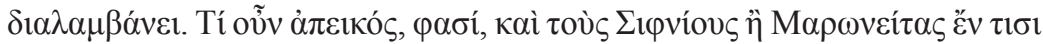

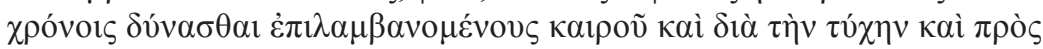

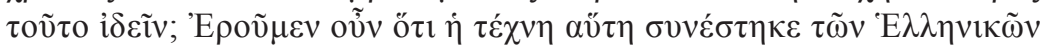

\footnotetext{
${ }^{17}$ Cf. le commentaire de Patillon, 2008b: p. 94 n. 3.

${ }^{18}$ Les deux espèces étaient cependant perçues comme étant si proches que l'on trouve, chez des rhéteurs latins, un échange: des auteurs présentaient comme exemple de l'invraisemblance ce que d'autres donnaient comme exemple de l'impossible (cf. Calboli Montefusco, 1986, p. 23).
} 


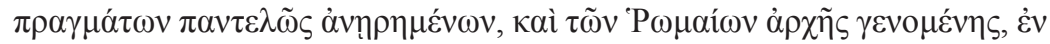

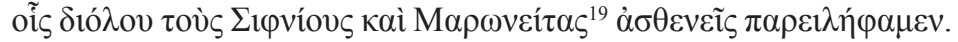

Certains critiquent cet exemple des Siphniens et des Marônites, disant que, même si ces villes sont insignifiantes et faibles pour réussir de tels exploits, il y a quand même selon Hérodote plusieurs villes qui sont devenues soit petites soit grandes, et aussi selon Thucydide, dans son histoire des Lacédémoniens. Qu'y a-t-il d'improbable - disent-ils - à ce que les Siphniens et les Marônites, à un certain moment, puissent saisir une occasion et, par un revers de la fortune, puissent viser ce but? Nous répondrons qu'il a composé ce traité à un moment où les états grecs avaient complètement disparu et qu'on était sous l'hégémonie romaine, et qu'à cette époque, avons-nous appris, Siphnos et Marônéia n'étaient que des nations totalement insignifiantes.

L'histoire est ce qu'elle est. Toutefois, elle aurait pu être autre chose.

Marcellinus (IV 161.26-162, Walz, 1833a), sur ce point, semble appartenir à la même tradition critique des devanciers de Sopatros: l'exemple des Siphniens et des Marônites, à son avis, est faible et mauvais; débattre sur le dessein d'hégémonie de ces peuples n'est pas impossible, comme le démontre le fait que, d'après Thucydide, Mycènes étant un petit état a joué un rôle prééminent.

L'Anonyme P, cependant, voit une différence importante entre les deux espèces (2.83.2-5, Duarte, 2006: = VII 150.9-13, Walz, 1832-1836): l'invraisemblable se base sur la qualité de la personne, l'impossible sur la nature de l'acte. Et il mène une critique assez tranchante à Hermogène (ib. 2.83.7-10 = VII 150.17-23, Walz, 1832-1836), jugeant que le rhéteur même s'est rendu compte de s'être mis lui-même dans un piège, essayant ensuite de corriger son tort:

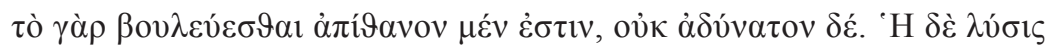

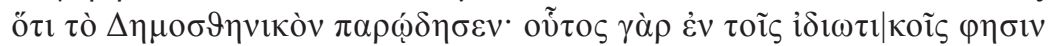

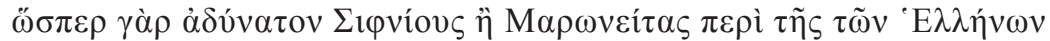

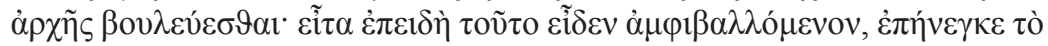

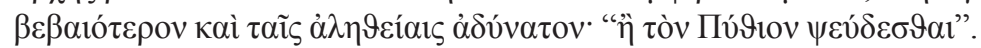

Former un dessein, c'est invraisemblable, non pas impossible. La solution est qu'il a parodié Démosthène. En effet, celui-ci, dans ses discours sur des sujets privés, dit comment il était impossible que les Siphniens ou les Marônites forment le dessein de commander aux Grecs. Puis, le technographe, se rendant compte de l'ambigüité de cet exemple, ajoute une déclaration plus sûre et vraiment impossible: « ou que le Pythien dit des mensonges. ${ }^{20}$

Les passages de Démosthène en cause sont Organisation financière 13.34 et Traité avec Alexandre 17.23 .

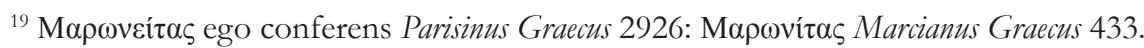

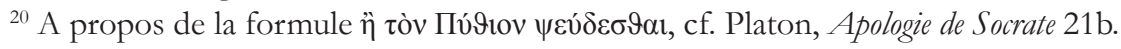


Suite aux questions inconsistantes, Hermogène présente une autre série comportant

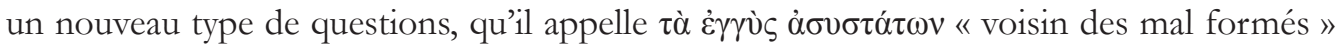
(ib. I 22-24). Ces sont là des questions qui, contrairement aux mal formées, peuvent sous certaines conditions être mises en examen. Examinons la deuxième (ib. I 23):

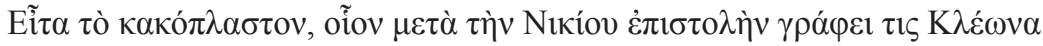

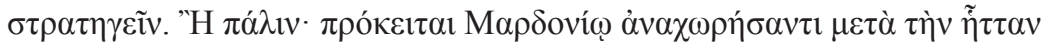

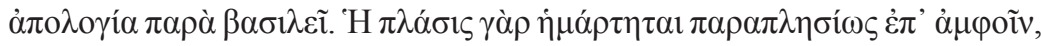

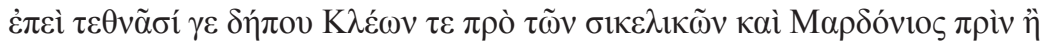

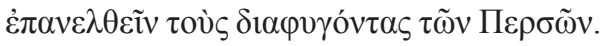

Ensuite la question mal imaginée: suite à la lettre de Nicias quelqu'un propose Cléon comme stratège; ou bien encore Mardonios de retour au pays après sa défaite doit présenter sa défense devant le Grand Roi: la fiction est pareillement fautive dans les deux cas, puisque, on le sait, Cléon est mort avant les évènements de Sicile et Mardonios avant le retour des Perses rescapés.

Le problème a suscité des commentaires chez des scoliastes d'Hermogène. Examinons les données de l'histoire: le premier cas est mal conçu à cause de son caractère anachronique: la lettre de Nicias date de 414 av. J.-C., du temps des évènements de Sicile et Cléon était déjà mort en 422 à la bataille d'Amphipolis. ${ }^{21}$ Quant à Mardonios, il était mort au combat près de Platée, en 479, avant le retour des Perses rescapés. Sopatros (V 72.2021, Walz, 1833b) et le commentaire parallèle attribué à «Sopatros » dans le codex Parisinus

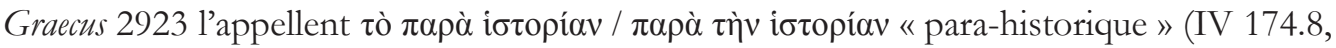

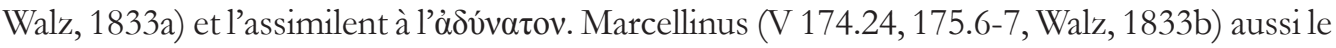
désigne ainsi. ${ }^{22}$ Les deux se définissent par rapport à l'histoire. ${ }^{23}$ En revanche, la mal imaginée n'est pas totalement dépourvue de ressources pour l'examen, mais la rhétorique censure au préalable ce à quoi il manque un minimum de correction historique et le refuse (Patillon 2008b: p. 95 n. 3). Les exemples de Sopatros (V 72.16-20, Walz, 1833b): si l'on conseillait d'envoyer Périclès comme stratège à Chéronée ou Démosthène comme ambassadeur chez les Péloponnésiens. Les impossibilités historiques: la bataille de Chéronée a été engagé le 338 av. J.-C. entre Philippe II de Macédoine et une coalition hellène, Périclès est décédé quatre-vingtonze ans avant; Démosthène a vécu entre 384 et 322, alors que la guerre du Péloponnèse a duré entre 431 et 304. Sopatros en conclut péremptoirement (V 72.20, Walz, 1833b): oű $\pi \omega$

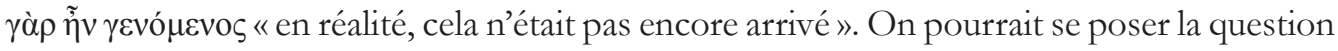

\footnotetext{
${ }^{21}$ Cf. Thucydide VII 10-13.

${ }^{22}$ Le latin Grillius aussi (Jakoby 9, 210-215) suit cette même tradition terminologique de désignation, mettant ainsi en évidence son caractère non-historique.

${ }^{23}$ L'Anonyme $\mathbf{P}$ précise que la différence entre ces deux types de question est le temps, parce que des personnes mortes figuraient dans la mal imaginée comme si elles étaient encore vivantes (cf. aussi Syr. R2 40.17-20), et que celle-ci n'était pas forgée comme si elle était naturellement impossible (Duarte, 2006, 3.92.2-4 = W7 158.9-12).
} 
de savoir pourquoi cette rhétorique aurait admis de tels types de problèmes, sachant qu'ils sont in limine litis à écarter de tout débat. A moins qu'on n’en puisse pas invoquer comme cause l'ignorance de l'histoire. Aristote, donc, aurait eu raison de dire que l'orateur est tenu de maîtriser une pléthore de domaines, parmi lesquels l'histoire.

L'histoire est une ressource dont la rhétorique peut se servir pour atteindre ses buts argumentatifs, et ceci au point de rendre les praticiens de l'éloquence dépendants des contraintes imposées par l'outil dont ils se servent et de les mettre dans l'impasse. Mais c'est là même, quand ce risque se présente, que la rhétorique renverse les choses. Voici que l'art et le génie l'emportent et que l'histoire redevient un outil, et pour les seuls buts de l'aiguisement des esprits et des raisonnements. C'est la troisième approche: si l'histoire impose des contraintes, on peut les laisser un peu tomber, les ignorer et poursuivre le débat.

Les orateurs attiques témoignent d'un usage des ressources fournies par l'histoire qui pouvait aller au point de la transformer, comme l'a démontré Nouhaud (1982). Il s'agissait là plutôt d'interprétations personnelles du passé récent aux fins persuasives.

Si l'on modifie certaines circonstances des évènements évoqués, on aura des questions bien formées. Si l'on échange les personnes, par exemple: si nous avions parlé des Lacédémoniens ou des Athéniens, au lieu des petits états de Siphnos et Marônéia, on aurait eu là une autre espèce de question, bien formée, dit Sopatros (V 68.22-28, Walz, 1833b), ou - selon l'exemple de «Sopatros » (IV 160.26-27, Walz 1833a) -, si on parlait du peuple de Pella, la capitale de la Macédoine. On emprunte à l'histoire un motif pour un discours de thème politique qui soit en accord avec les évènements historiques, mais il ne sera tenu que s'il ne s'heurte pas aux contraintes historiques. Celles-ci prennent le gouvernail à nouveau.

La dévalorisation de l'histoire est bien évidente dans la première espèce des questions

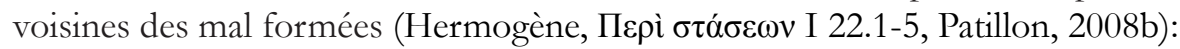

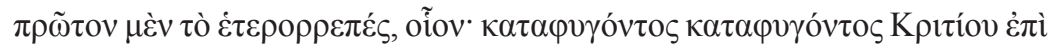

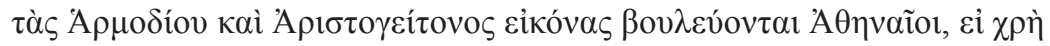

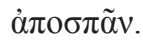

Premièrement la question déséquilibrée: Critias c'est réfugié auprès des statues d'Harmodios et d'Aristogiton et les Athéniens délibèrent pour savoir s'il faut l'en arracher.

Voici une version de l'histoire qui, comme dans les questions invraisemblable et impossible, infirme toute histoire. Rappelons les faits: Critias était l'un des «Trente Tyrans » qui ont gouverné Athènes suite à la défaite de la guerre du Péloponnèse en 404 av. J.-C. Il fut connu comme un instigateur de la terreur. Ce régime ne dura qu'une courte période de huit mois. Harmodios et Aristogiton furent, un siècle avant (514 av. J.-C.), les tueurs d'un autre tyran, Hipparchos. Par leur acte ils se sont fait une réputation d'héros et de symboles de liberté et de la démocratie. Un culte a été institué en leur honneur (cf. Fornara, 1970: 155 sq.). Voici la raison des statues. Quant à Critias, il est tombé à la bataille de Munichia au Pirée, entre les mains des partisans de la démocratie en exil (commandés par Thrasybule) et des supporters des Trente (cf. Xenophon, Hellenica 2.4.19). Cette bataille a précipité le 
régime vers sa fin. Critias a donc été frappé par la mort avant. Une version telle que celle proposée par Hermogène suppose, au contraire, que le tyran aurait pu être témoin de la restauration de la démocratie.

Hermogène, néanmoins, ne traite pas ce cas comme une question impossible, un thème qui aurait pu être rejeté du fait qu'il ne surmonte pas le test de l'histoire. Il peut sous certaines conditions avoir de la consistance. Les commentateurs du rhéteur de Tarse l'ont bien compris. Sopatros (V 71.27-72.16, Walz 1833b), par exemple, explique que la qualité de la personne accusée - dans le cas présent un tyran - a beau donner de la force à l'accusation, elle peut quand même faire appel au droit d'asile auprès des statues. Puis le commentateur s'égare un peu. Le réfugié alléguant qu'on ne peut pas prétendre que la démocratie prévale si on abroge la loi qu'elle même a approuvée, pourtant cette loi est quand même un peu faible, car un tyran n'aurait pas dû avoir le droit de refuge auprès des statues de tyrannicides, puisque, s'ils étaient encore vivants, ils l'auraient combattu. Le thème peut être discuté dans ses cohérences et incohérences strictement rhétoriques, visant l'établissement d'arguments, de sorte que toutes les parties aient des preuves fortes, crédibles et différentes, selon les

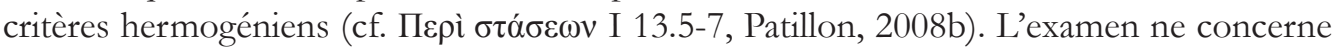
plus l'évènementiel, il ne s'agit donc pas de purement et simplement savoir si l'histoire a été ou non respectée.

Revenons au problème mal imaginé. Sopatros (W5 72.22-28, Walz 1833b) nous dit qu'il existe une condition dans laquelle il peut être résolu et déclamé, si para-historique qu'il soit. C'est qu'on l'énonce comme une version alternative de l'histoire, à titre hypothétique

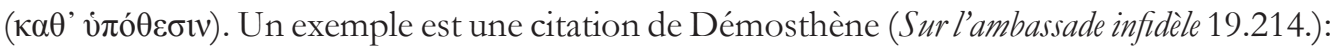

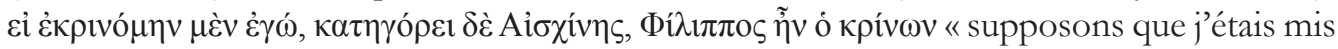

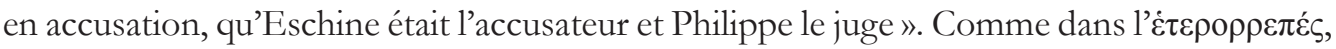
l'histoire peut faire l'objet de fiction, les données de l'histoire ne servant que des prétextes, des pions à manipuler dans le jeu de l'éloquence.

Plus que de se servir de l'histoire (la première approche) comme des exemples pour soutenir les arguments, par manipulations nous voulons dire qu'il s'agit de passer à côté des contraintes de l'histoire et d'engager le débat. L'histoire, ainsi que les récits mythiques, constituent alors un thesaurus de thèmes à explorer dans des exercices d'éloquence, autant que l'art et ses ressources le permettent. C'est bien une façon de procéder de la rhétorique en sophistopolis, comme la désigne D. A. Russel (1983).

\section{Conclusion}

On vient d'étudier quelques aspects des rapports entre rhétorique et histoire. Elles étaient deux discours et aussi deux mondes éloignés, l'un n'ayant rien à voir avec l'autre.

Si dans le discours rhétorique on émet plutôt une opinion dont il faut convaincre quelqu'un, en revanche l'histoire serait plutôt objective, elle viserait à relater des évènements. Comme Hérodote l'a exprimé dans la préface de son œuvre (I, 1) l'objet de l'histoire est la préservation de la mémoire de faits accomplis par les hommes ainsi que leur causes ( $\tau \grave{\alpha}$ 


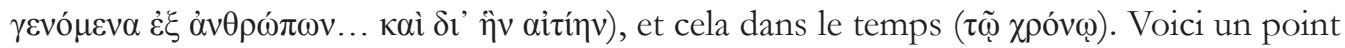
où la rhétorique, dans certaines de ses tendances et métamorphoses, vise ailleurs. En effet, elle s'occupe d'affaires hors de tout temps et espace, au moins du temps et de l'espace des hommes concrets et historiquement définis, en se créant un univers parallèle avec ses personnages, lois, débats, affaires et thèmes à lui (dans le sens où Russell, 1983, l'a compris et décrit).

Pourtant, les choses ne sauraient pas être si strictes. Un examen plus précis des choses a pu déceler certains points de contact entre elles. La rhétorique fournit et entraîne les compétences de composition littéraire requises pour le métier d'historien (qu'on se rappelle les déclarations de Aélion Théon). L'histoire, en revanche, fournit à l'orateur que ce soit au barreau d'un tribunal, dans une assemblée ou en n'importe quelle occasion

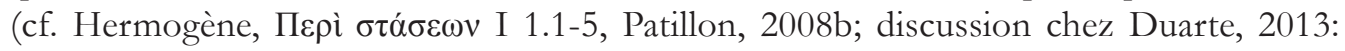
p. 207-223) - des exemples, thèmes et ressources pour les arguments à construire. Et ceci, même au prix de l'altération des données de l'histoire, car il n'y est point une historia gratia bistoriae. Bref, ce sont là surtout des apports et rapports de type utilitaire, où chacun de deux arts sert à la fois les propos de l'autre.

\section{BibliographiE}

ALEXANDRE JÚNIOR, Manuel. Importância da cria na cultura helenística. Euphrosyne, 17, p. 31-62, 1989.

CALBOLI MONTEFUSCO, Lucia. La dottrina degli status nella retorica greca e romana. Hildesheim: Olms, 1986.

DUARTE, Rui Miguel. Comentários ao tratado sobre os Estados de causa de Hermógenes de Tarso por autor anónimo. Aveiro: Universidade de Aveiro, 2006.

DUARTE, Rui Miguel. «Quelle est l'étendue de la rhétorique selon Hermogène? Dans les assemblées, les tribunaux et... partout». Euphrosyne, v. 41, p. 207-223, 2013.

FELTEN, Nicolaus. Nicolai Progymnasmata. Leipzig: Teubner, 1913.

HOCK, Ronald F; O’NEILL, Edward N. The Chreia in Ancient Rhetoric, v. I: The Progymnasmata. Atlanta, Georgia: Scholars Press, 1986.

FORNARA, C. W. «The cult of Harmodios and Aristogiton ». Philologus, v. 114, p. 155-80, 1970.

KENNEDY, George A. Progymnasmata. Greek Textbooks on Prose Composition and Rhetoric. Atlanta, GA: Society of Biblical Literature, 2003.

LAUSBERG, Heinrich. Handbuch der literarischen Rhetorik. Munich: Max Hüber, 1960.

NOUHAUD, Michel. L'utilisation de l'histoire par les orateurs antiques. Paris: Les Belles Lettres, 1982. 
PATILLON, Michel. La théorie du discours chez Hermogène le rhéteur. Essai sur la structure de la rhétorique ancienne. Paris: Les Belles Lettres, 1988.

PATILLON, Michel. Aelius Théon. Progymnasmata. Paris: Les Belles Lettres, 1997.

PATILLON, Michel. La théorie du discours chez. Hermogène le rhéteur. Essai sur les structures linguistiques de la rhétorique ancienne. Paris: Les Belles Lettres, 1998.

PATILLON, Michel. Longin, Fragments; Rufus, Art rhétorique. Paris: Les Belles Lettres, 2001.

PATILLON, Michel. Pseudo-Aélius Aristide, Arts rhétorique I. Paris: Les Belles Lettres, 2002a

PATILLON, Michel. Pseudo-Aélius Aristide, Arts rhétorique II. Paris: Les Belles Lettres, $2002 \mathrm{~b}$.

PATILLON, Michel. Corpus rhetoricum I: Anonyme, Préambule à la rhétorique; Aphthonios, Progymnasmata; Pseudo-Hermogène, Progymnasmata. Paris: Les Belles Lettres, 2008 a.

PATILLON, Michel, Corpus rhetoricum II: Hermogène, Les états de cause. Paris: Les Belles Lettres, 2008b.

PATILLON, Michel. Corpus rhetoricum III 1: Pseudo-Hermogène, L'invention. Anonyme, synopses des exordes. Paris: Les Belles Lettres, 2012a.

PATILLON, Michel. Corpus rhetoricum III 2: Anonyme, scolies au traité Sur l'invention du PseudoHermogène. Paris: Les Belles Lettres, 2012b.

PATILLON, Michel. Corpus rhetoricum IV 1: Prolégomènes au De ideis. Hermogène, Les catégories stylistiques du discours (De ideis). Synopses des exposés sur les ideai. Paris: Les Belles Lettres, 2012c.

RABE, Hugo. Rhetores Graeci II. Syriani in Hermogem commentaria. Leipzig: Teubner, 1893 réimpr. 1985.

RUSSELL, D. A. Greek Declamation. Cambridge: Cambridge University Press, 1983.

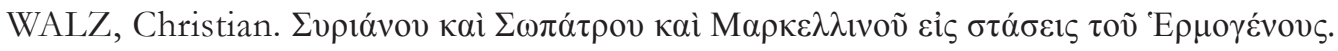
Stuttgart \& Tübingen: J. C. Cottae, 1833a, p. 39-846.

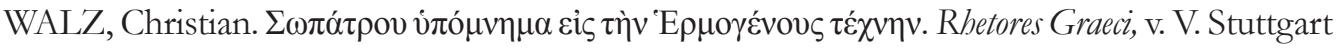
\& Tübingen: J. C. Cottae, 1833b, p. 1-211.

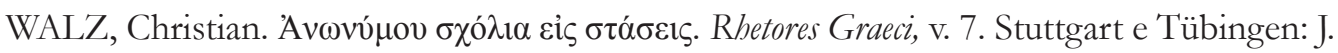
C. Cottae, 1832-1836, p. 104-696.

WOERTHER, Frédérique. Hermagoras. Fragments et Témoignages. Paris: Les Belles Lettres, 2012.

Recebido em: 20 de março de 2016

Aprovado em: 19 de julho de 2016 